\title{
Seeing in super-resolution
}

The 2014 Nobel Prize in Chemistry has been awarded in recognition of super-resolution fluorescence microscopy. To fully realize the tremendous promise of these methods for biological discovery, there are still challenges to be overcome.

We at Nature Methods need no convincing of the importance of methods for biological research. We are nevertheless delighted to note that in awarding this year's Nobel in Chemistry to scientists behind super-resolution fluorescence microscopy, the Nobel Committee has again honored methods developers. In the past 30 years, as a matter of fact, roughly a third of the prizes in Chemistry have been given for methods development. In Physiology or Medicine, this number is a little smaller but still substantial-six or seven prizes, depending how you count them, over the same period.

When we chose super-resolution microscopy (SRM) as our Method of the Year 2008, these techniques were at a peculiar phase in their development. The two main approaches-super-resolution based on stimulated emission depletion (STED) or on single-molecule localization (PALM, STORM and their variations) - had both been published: in the case of STED, already several years earlier. Structured illumination microscopy, which enables a twofold improvement in resolution over that of diffractionlimited imaging, had also been described. These methods were by no means in wide use by biologists yet. But we were convinced, and hopeful, that they were ripe for biological application.

And indeed they were. Today all the major superresolution approaches are part of commercially available setups. They have been shown to be applicable dynamically, in three dimensions, to living cells. They provide a finer view into subcellular structure, either alone or in correlation with electron microscopy, in fixed samples too. SRM of one or another flavor has been applied, for example, to image dendritic spines, to identify unexpected actin-spectrin lattice structure in axons, to obtain a detailed three-dimensional understanding of focal adhesion complexes, to study surface-receptor patterns and vesicular trafficking, and to examine chromosome segregation in bacteria.

That said, putting SRM to work for biology is far from easy. In addition to technical considerations that hold for any imaging approach - such as avoiding perturbation of a structure due to labeling, or minimizing phototoxicity-the nature of SRM methods brings up additional issues. For instance, efficient labeling of target structures is essential to obtain high-quality images and can pose a substantial challenge.

Applying SRM to biology even requires a shift in thinking. Not only do these approaches give a new view of cellular and macromolecular structuresrendering structures visible that could previously not be seen by light microscopy-but for localizationbased SRM, the data are fundamentally different from those produced by traditional imaging methods. Images based on single-molecule localizations are complex reconstructions of vast amounts of data. Practitioners must become accustomed to this new view of biological systems and learn how to make sense of this type of data.

Reconstructing a super-resolved image requires that single molecules are accurately localized and that these localizations are then put together to give a picture that corresponds to reality, with all the complications of fluorophore blinking, movement and low signal-to-noise ratio, among others, that are inherent to this regime and that must be taken into account for proper interpretation. A lack of sufficient care and expertise in data analysis can lead to low-quality images or even to artifacts-to seeing clusters, for example, where none exist.

Most laboratories carrying out biological studies using SRM include scientists able to implement the statistical handling of large data sets, typically with a background or at least some training in mathematics or physics. In this regard, SRM is one of many technologies driving biology, and biologists, toward a more quantitative approach.

Close and ongoing collaboration is vital between those who have defined the questions in their field, typically using more traditional biochemical and imaging methods, and those applying SRM to probe these questions more quantitatively. Together with technological improvements extending the current limits of resolution, speed, depth and color multiplicity, such collaboration will be an important aspect of bringing the biological application of super-resolution methods to its full fruition. 